\title{
Secure Management of Networked Batteries for Building Integrated Photovoltaics (BIPV) Systems
}

\author{
Fengling Han ${ }^{1}$, Xun $\mathrm{Yi}^{1}$, Rebecca Yang ${ }^{2}$, Ron Wakefield ${ }^{2}$, Yong Feng ${ }^{3, *}$ \\ ${ }^{1}$ School of Science, RMIT University, 124 La Trobe Street, Melbourne, VIC, 3001, Australia \\ ${ }^{2}$ School of Property Construction and Project Management, RMIT University, 124 La Trobe Street, Melbourne, VIC 3001, \\ Australia \\ ${ }^{3}$ Department of Electrical Engineering, Harbin Institute of Technology, 192 XiDazhi Street, Heilongjiang, 150006, China \\ *Corresponding author: E-mail: yfeng@hit.edu.cn
}

DOI: $10.5185 /$ amlett.2020.021469

This letter reviews the related research on Building Integrated Photovoltaics (BIPV) for efficient utilization of solar energy, and further clarifies the concept of networked batteries. Taking Lithium-ion batteries as an example, maximizing the energy storage is the target, which is achieved by managing the charging and discharging of the individual batteries within a network. With the optimal management of the networked batteries, buildings constructed by using PV-capable materials are expected to be energy self-sufficient, which leads to zero-carbon energy supply. It is pointed out that the real-time estimation on the State-of-Charge (SoC) of Lithium-ion battery is crucial for secure management of the networked batteries.

\section{Introduction}

Global warming, extreme weather and bushfire are on the top news towards the end of 2019 in Australia. On the other hand, energy shortage and population growth in Australia pose a challenge for reliable electricity supply to homes and business consumers, particularly during the peak electricity demanding period. There is a high cost for increasing the electric power network capacity due to the infrastructure investment required. In addition, there is $10 \%-15 \%$ associated transmission loss for distributing the electricity to the loads [1]. For example, installing an additional 5TWh electricity generation capacity will cost around \$6 trillion, with further transmission and distribution costs of $\$ 10$ trillion. The power stations have become unprofitable as evident by the closure of numerous power plants in Australia in recent years [2]. Renewable energy is considered as a cost-effective solution for both providing power to businesses and residents and addressing peak electricity demanding issues.

Building Integrated Photovoltaics (BIPV) is a technology that integrates PV panels into building construction. Solar energy generated on Earth is far in excess of global energy requirements [3]. Design and construct buildings that uses PV-capable material has attracted significant amount of interest from researchers, engineers and manufactures alike. The solar energy could be harvested at buildings by the following two ways [4]:

- Solar thermal collectors: transform solar radiation into heat by using parabolic mirror arrays, and

- Photovoltaic (PV) panels: produce electricity from solar radiation by using semiconductor materials.

Integration of PV panels with building materials could provide $100 \%$ renewable energy to the electric loads within the buildings [5-7], which leads to a zero-carbon energy supply ecosystem. Power grid operators assume the load as a given and adjust the supply accordingly. To provide reliable and stable clean electricity to power the loads, however, the solar energy generated should exceed the amount of demand. Due to the intermittency of PV systems, which means that the generation of solar energy (termed as variable renewable energy) is highly dependent on the weather condition. Therefore, the solar energy needs to be stored (and further processed) once being generated.

For providing zero-carbon clean energy to power electric loads, energy storage systems should be in paired with the PV panels. This letter reviews self-sufficient electric power for buildings based on BIPV technology. BIPV-based zerocarbon ecosystem architecture, energy storage systems and monitoring the internal states of individual batteries are reviewed. Paired with PV-capable materials, the storage batteries are connected into a network for maximizing the energy storage and optimizing the system performance. The observer technology is used for estimating the state-ofcharge (SoC) of individual batteries, based on which secure management and performance optimization of the BIPV electrification could apply. We propose a secure management of networked batteries for: 1) maximizing the solar energy storage, 2) optimizing the stored energy utilization. The two targets will be achieved by coordinating the charging and discharging of individual batteries. Real-time monitoring the internal parameters of rechargeable batteries is the key.

\section{BIPV-based zero-carbon power supply ecosystem}

Investigation shows that $40 \%$ of the worldwide energy demand is consumed by buildings $[\mathbf{5 , 1 5}]$. BIPV systems generate electricity at the point of being consumed, which 


\section{Advanced Materials Letters www. vbripress.com/aml}

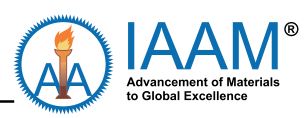

www.iaamonline.com eliminate electricity transmission and distribution as well as their associated losses, as a result, BIPV systems reduce capital and maintenance costs for power supply utilities. This fact is appealing to reliability and cost effectiveness of power supply.

The electromagnetic radiation emitted by the Sun could be captured from buildings by 1) tile-style modules, skylights and shingles on building roof, and 2) façade such as curtain wall, spandrel panels and glazing [4-7]. The harvested energy from roof or façade will potentially create a zero-carbon building. Aligning with this goal, increasing the net power output, collecting more solar energy, BIPV electrification have been widely investigated.

\section{Increase the net power}

Zero-carbon power supply motives researchers to explore efficient methods in BIPV applications. Khan etc. have conducted extensive experimental study on reflecting the Sun light into PV module [15]. An amplified output power is achieved by increasing the sunlight flux intensity per unit area on the BIPV.

The hybrid photovoltaic and energy storage systems have been investigated to facilitate the low-voltage grid [16-18]. In these researches, the system block diagram, PV data and consumer load data are presented to demonstrate the techno-economic analysis. The charging and discharging of the battery are operated optimally to minimise a pre-defined cost function. A dual battery system is further proposed for off-grid applications in [17], which works for charging and discharging the batteries alternatively.

\section{Connection mode of PV generated solar energy}

The energy generated in the BIPV systems is decentralized, besides harvesting energy, well-integration is the key for zero-carbon buildings. The harvested energy could be configured as either grid-connected or off-grid. Grid-connected configuration could further be categorized into centralized model and distributed model [9-11].

Majority of the existing power energy management focuses on integration of PV to supplement the centralized power networks, as shown in Fig. 1 [12]. Alternative Current (AC) mode has been used as the electric power supply for decades. As a result, the electricity wires in the power transmission and distribution carry AC. Therefore, a grid-connected PV inverter is used to transform the Direct Current (DC) power (direct generated from PV panels) into AC power, which will be then fed into existing power networks. The PV generated solar energy in the gridconnected mode is mainly used for saving electricity bills, load shifting, and demand response [10].

The solar energy generated by PV panels could not be used for driving load directly. Off-grid BIPV systems, in some cases is termed as a micro-grid, require paired battery storage. This is the case of zero-carbon buildings. Power energy management is required to coordinate the charging and discharging of storage batteries by storing the generated energy (charge) when solar irradiance is available; and supplying the energy (discharge) to loads inside the buildings when needed.

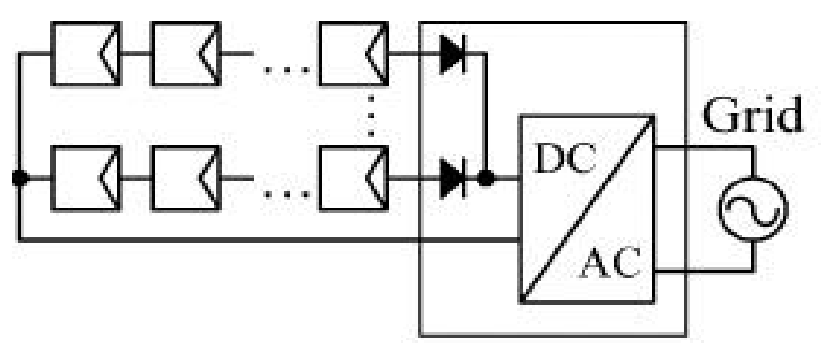

Fig. 1. Centralized configuration of BIPV systems.

\section{BIPV systems electrification}

With the increase number of DC load, for example, LED, IT equipment, consumer electronics, etc., at commercial properties and residential homes, BIPV electric systems prefer adopting DC instead of AC [6]. The rational of this preference is that the energy generated from solar panels is in the form of DC, energy stored in battery is DC. Further, the majority of loads are DC $[\mathbf{6 , 1 3}]$.

Alleviating the AC/DC and DC/AD conversion will greatly increase the reliability and efficiency of BIPV electrification. Low Voltage DC (LVDC) grid is highly recommended for BIPV electric architecture [12], due to:

- LVDC is compatible with the DC load, which leads to increase the efficiency;

- LVDC system simplified the conversion, which results in better reliability;

- Both input/output are DC power, which alleviates the AC components.

LVDC grid is composed of solar panels and modules (cluster of the panels) as the energy sources, battery storage, load and monitoring devices, as shown in Fig. 2.

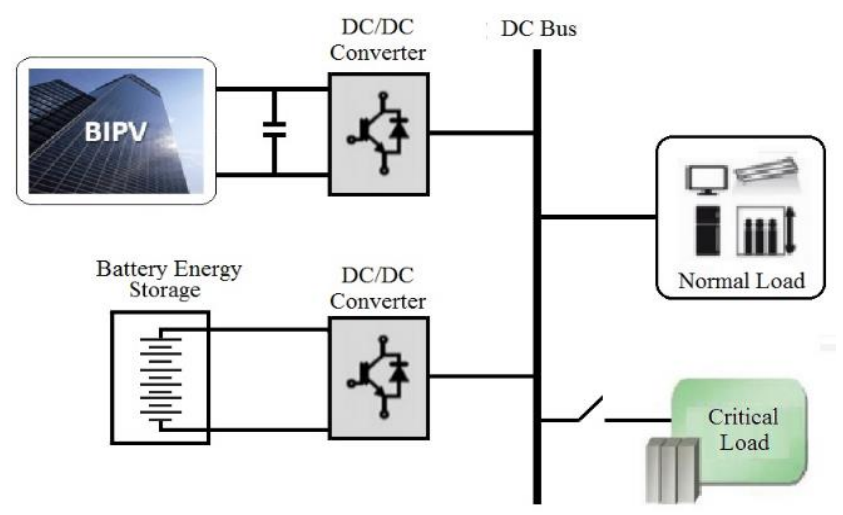

Fig.2. Self-sufficient electrification of BIPV systems.

\section{Energy storage systems (ESSs)}

Due to the highly unpredictable and uncontrollable nature of solar energy sources, the produced electric energy is unbale to satisfy the requirement of loads. Energy storage systems (ESSs) is employed to function as a buffer [18,19]. The electrical energy generated from PV panels can be stored directly in electrical form or converted and stored in mechanical and electro-chemical energy storage systems. 


\section{Advanced Materials Letters www. vbripress.com/aml}

Advanced energy management technology enables converting the intermittent solar energy to the quality of powering the load, thus, provides cost effective, stable and flexible zero-carbon ecosystems.

\section{Rechargeable batteries for energy storage}

Generally speaking, batteries store electrical energy generated from renewable sources include: Lead-acid, Nickel-cadmium, Sodium Sulphur, Lithium-ion (Li-ion), and flow batteries, Zinc bromine, and polysulfide bromine [11]. Each type of battery has advantages and disadvantages in terms of power rating, discharge duration, response time, power and energy densities, efficiency, lifetime, etc. Due to the superior properties of high energy density, light weight, no memory effect and less self-discharge, Li-ion battery technologies have dominated the market for electricity energy storage [20]. Battery management systems (BMS), include hardware and software, monitor and control the charge and discharge process of the battery pack, maintain the cells in a pack work in the safe operating area and extending their life expectation. However, as the electrolyte material is flammable, better safety and reliability of Li-ion batteries and the improvement on performance is still a challenge.

\section{Battery-supercapacitor hybrid energy storage}

For BIPV systems, power demand comprises of both static and dynamic loads. Supercapacitor, also known as electric double layer capacitors, has fast response than batteries, which could enhance the capacity of powering the dynamic loads $[\mathbf{8 , 2 1}]$. Battery-supercapacitor hybrid storage has been reviewed comprehensively in [21], which covers coordinating and powering both DC and AC loads with matched supply-demand, decoupled load profile and control strategies in a hybrid AC-DC micro-grid.

\section{Monitoring the internal state of batteries}

Take Li-ion battery as an example, inside a battery pack, the electrochemical cells are connected in series to increase the operating voltage and in parallel to increase the available current. With the progress of battery packs being in service, the cell capacity degrades as they are repeatedly being charged and discharged. Monitoring capacity loss is critical for managing the batteries to work in safe operation area [22].

\section{Modelling a battery cell}

The State-of-Charge (SoC) describes the present battery capacity in percentage with the maximum capacity. When service as a power supply, SoC represents the available energy at a prescribed discharge rate [23]. Under normal charging conditions, the terminal voltage of a cell increases with the level of charge; this feature is used as an indicator of SoC by electronic engineers. However, SoC has a time varying nonlinear characteristics, and the parameters of electrochemical cell vary with respect to operating region/ environment. The task of battery management systems includes cell protection, charge control, dynamic cell balancing (during charging and discharging), SoC and State-of-Health ( $\mathrm{SoH}$ ) estimation, logbook (the history), communication. Equivalent circuit model of battery, shown in Fig. 3, is derived for better description on the internal state of individual Li-ion cells.

In Fig. 3, $R_{\mathrm{S}}$ represents the thermodynamic properties of the battery chemistry. The nominal capacity open-circuit voltage (OCV) is a function of state-of-charge (SoC); and $R_{\mathrm{p}}, C_{\mathrm{p}}$ represent the internal kinetic of the cell impedance.

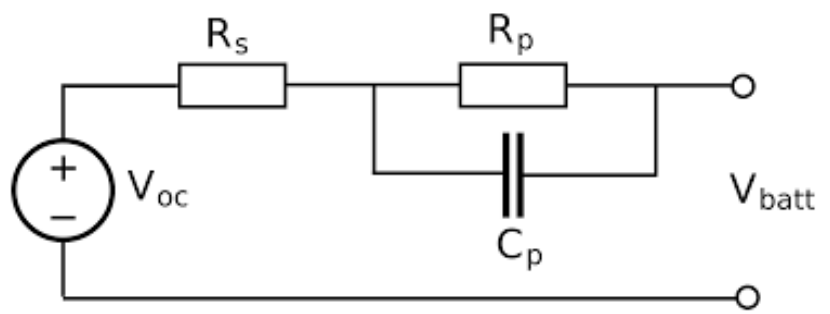

(a)

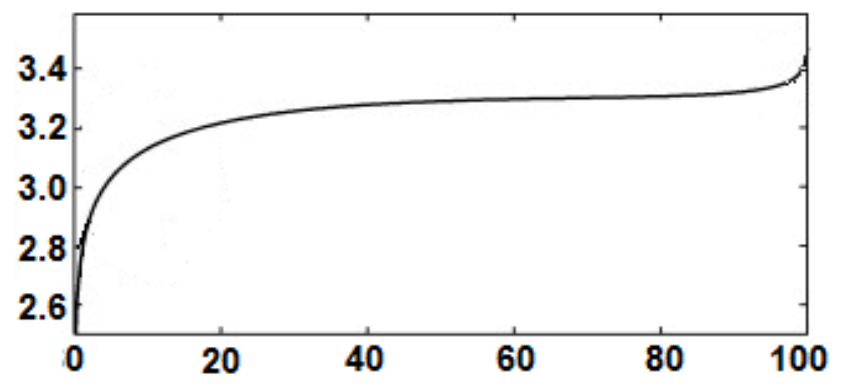

(b)

Fig.3. (a) Equivalent circuit model of a Li-ion cell. (b) Charging of the Liion cell (voltage in volt).

\section{Monitoring the state-of-charge of a cell}

For safe operation, the parameters variation inside the cells needs to be monitored. However, the battery packs are sealed for safety reasons when manufacturing, such as fireproof. As a result, the internal parameters of a battery cells are inaccessible once they are manufactured.

For estimating the internal parameters, the influence of voltage and current transducer measurement uncertainty has been analysed $[\mathbf{2 2 , 2 3}]$, the results show that both fixed error and random error from the sensor affect the SoC estimation.

The Kalman filter (KF) technology has been used to estimate the internal states of rechargeable batteries, however, it applies when the noise is within the system bandwidth [25]. Therefore, they could not provide accurate estimation to the internal state of batteries in the practical environments. In addition, the complexity of internal parameters variation and the complex dynamics process of batteries, compound with parameter bias during the manufacture process, all these making reliable SoC estimation difficult.

Observer technology has been used to establish a simulated model, which adopts the same input and output of the working batteries for estimating the internal parameters of Li-ion battery [26]. Due to the limited number of parameters available, a cascade method has been 


\section{Advanced Materials Letters www. vbripress.com/aml}

proposed for monitoring the SoC of Li-ion battery. The dynamic stress test (DST) and federal Urban Driving Schedule (FUDS) have been conducted to prove the effectiveness of the proposed method. DST is designed by the US Advanced Battery Consortium (USABC) [27]. It allows the verification of whether a battery can deliver a specified power to consumers under all anticipated operating conditions. FUDS is a commonly used simulation available to represent the power demands. All experimental data are captured from LNMC/Graphite Li-ion cells in the Arbin BT2000 battery test system [28]. The basic specifications of test samples (INR 18650-20R) are shown in Table 1. In reference to the data by DST test, the parameters $R_{s}, C_{p}$, and $R_{p}$ are estimated using the Recursive Least Square (RLS) algorithm. All tests were conducted for $80 \%$ battery capacity level at $25^{\circ} \mathrm{C}$. The battery has a shape of cylinder, with the length of $64.85 \pm 0.15 \mathrm{~mm}$, and diameter of $18.33 \pm 0.07 \mathrm{~mm}$. Weight 45 gram.

Table 1. Experiment condition.

\begin{tabular}{l|c}
\hline Battery (Parameters) & Specifications (Value) \\
\hline Capacity Rating & $2000 \mathrm{mAh}$ \\
\hline Cell Chemistry & Graphite/LiNiMnCoO2 (NMC) \\
\hline Weight & $45.0 \mathrm{~g}$ \\
\hline Dimensions (mm) & $18.33 \pm 0.07 \mathrm{~mm}$ \\
\hline Length & $64.85 \pm 0.15 \mathrm{~mm}$ \\
\hline Nominal voltage & $3.6 \mathrm{~V}$ \\
\hline Maximum current & $22 \mathrm{~A}\left(\mathrm{at} 25^{\circ} \mathrm{C}\right)$ \\
\hline Upper/lower cut-off voltage & $2.5 \mathrm{~V} / 4.2 \mathrm{~V}$ \\
\hline Usage temperature & $0-50^{\circ} \mathrm{C}$ \\
\hline
\end{tabular}

(a)

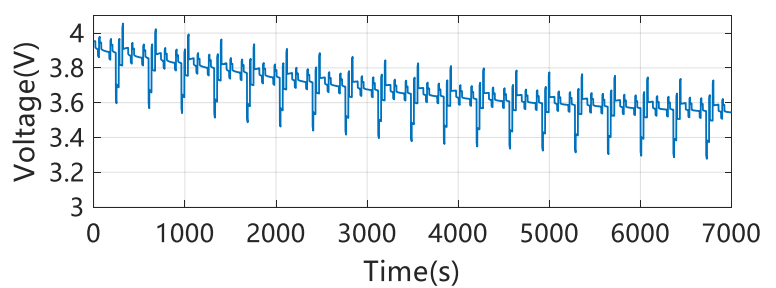

(b)

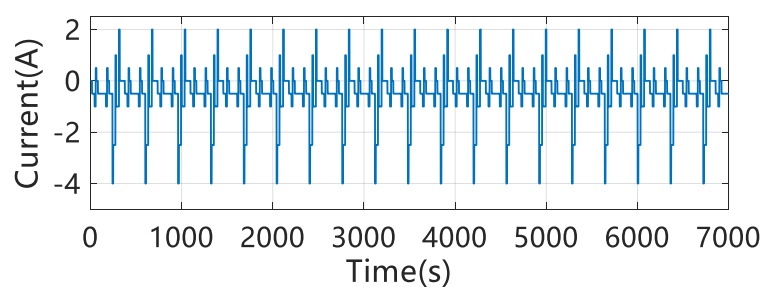

(c)

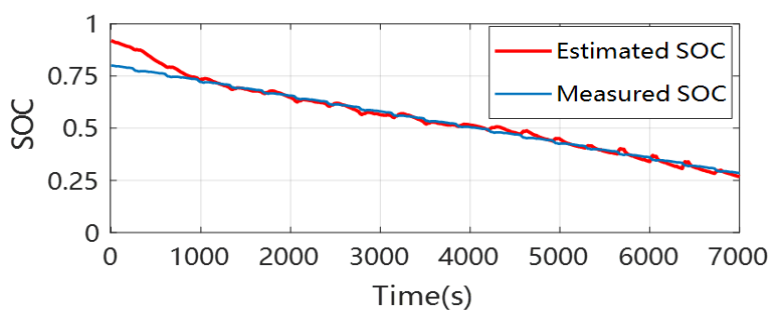

Fig. 4. (a) Measured voltage, (b) Measured current; and (c) Measured SoC of NMC cell via DST at $25^{\circ} \mathrm{C}$.

The measured current and terminal voltage of an INR 18650-20R cell from DST are shown in Figs. 4(a) and 4(b). The true value of $\mathrm{SoC}$, its estimation results using the sliding-mode observer are depicted in Fig. 4(c). It can be seen that the SoC is estimated accurately. The measured current and terminal voltage of another INR 18650-20R cell from FUDS are shown in Figs. 5(a) and 5(b). Its SoC estimation results is depicted in Fig. 5(c). It can be seen that the sliding-mode observer has good estimation precision and faster dynamical response in FUDS condition.

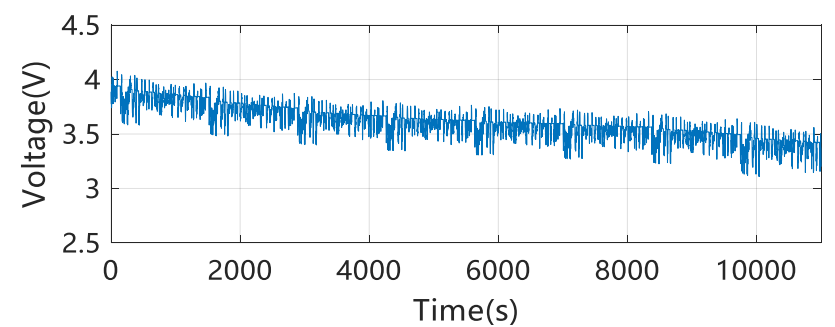

(a)

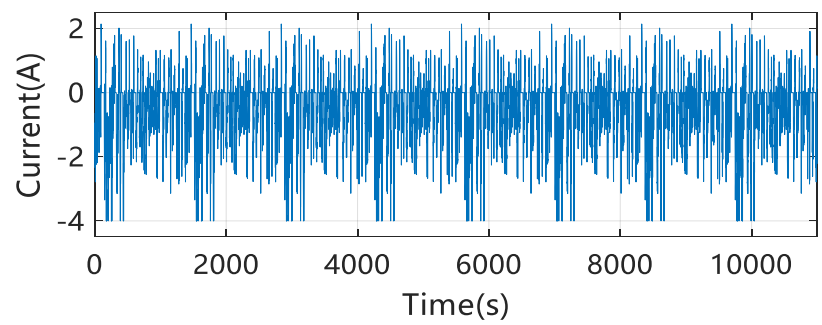

(b)

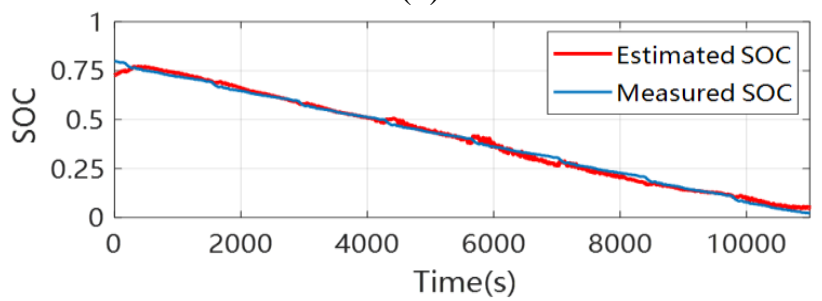

(c)

Fig. 5. (a) Measured voltage, (b) Measured current; and (c) Measured SoC of NMC cell via FUDS at $25^{\circ} \mathrm{C}$.

\section{Conclusion and future perspectives}

This letter reviewed the electric energy architecture and management for BIPV systems. The Low Voltage DC model is recommended for BIPV electrification. For achieving self-sufficient power, battery storage needs to be paired with the PV panels. Connecting the storage batteries into a network, sharing and secure managing the renewable energy will create a zero-carbon energy ecosystem. Maximizing the renewable energy storage and efficient use of collective renewable energy resource could be achieved by controlling the charge and discharge of individual storage batteries in a dynamic networked environment.

Future work should focus on the detail architecture of the network topology, considering the hybrid load of both DC and $\mathrm{AC}$.

\section{Acknowledgements}

The authors would like to acknowledgements Mr. Chen Xue for his help in providing the simulation figures. This research is supported by National Natural Science Foundation of China (61673132), Australia Research Council of Australia (LP180101062), and RMIT University funding (EOF-TI007). 


\section{Advanced}

\section{Materials Letters}

\section{www. vbripress.com/aml}

\section{Keywords}

BIPV, networked batteries, solar energy, zero-carbon ecosystem.

\section{Received: 01 November 2019}

Revised: 24 December 2019

Accepted: 31 December 2019

\section{References}

1. Casten, T.; Downes, B; Skeptical Inquirer, 2005, 29

2. Blakers, A.; Lu, B.; Stocks, M.; Australia Clean Energy Council Report 2016.

3. Lewis, N. S; Science, 2005, 315, 798.

4. Ravyts, S.; Vecchia, M.D.; Broeck, G.; Driesen, J.; Energies, 2019, 12, 1251.

5. Heinstein, P.; Ballif, C.; Perret-Aebi, L.; Green, 2013, 3, 125.

6. Biyik, E.; Araz, M.; Hepbasli, A.; Engineering Science and Technology, 2017, 20, 833.

7. Debbarma, M.; Sudhakar, K.; Baredar, P.; Renewable and Sustainable Energy Reviews, 2017, 73, 1276.

8. Krishan, O.; Suhag, S.; Int. J. Energy Res. 2019, 43, 6171.

9. Li, C.; Wang, R.Z.; Renewable and Sustainable Energy Reviews, 2012, 16, 6191.

10. Ogunniyi, E. O.; Pienaar, H.: Proc. Int. Conf. Domestic Use of Energy, Cape Town, South Africa, 2017, 233.

11. Krishan, O.; Suhag, S.; Int. J. of Energy Research, 2019, 43.

12. Liu, B.; Duan, S.; Cai, T.; IEEE Trans. On Power Electronics, 2011, 26, 1418 .

13. Arunkumar, G.; Elangovan, D.; Sanjeevikumar, P.; Energies, 2019, 12, 2157.

14. Hu, J; Harmsen, R; Graus, W.C; Worrell, E; Broek, van M; Renewable and Sustainable Energy Reviews, 2018, 81, 2181.

15. Khan, M.A.; Zeb, K.; Uddin, W.; Electronics, 2018, 7, 119.

16. Khan, M.A.; Zeb, K.; Sathishkumar, P.; Electronics, 2018, 7, 63.

17. Nengroo, S.H.; Kamran, M.A.; Ali, M.U.; Electronics, 2018, 7, 177.

18. Ali, M.U Kamran, M.A.; Kumar, P.S.; Energies, 2018, 11, 2940.

19. Nengroo, S.H.; Ali, M.U.; Zafar, A.; Electronics, 2019, 8, 176.

20. https://apvi.org.au/building-integrated-pv-bipv-best-practiceguidelines/

21. Khalid, M.; Energies, 2019, 12, 4559.

22. Ali, M.U.; Zafar, A.; Nengroo, S.H.; Energies, 2019, 12, 446.

23. Ali, M.U.; Zafar, A.; Nengroo, S.H.; Electronics, 2019, 8, 709.

24. Chaturvedi, N. A.; Klein, R.; Christensen, J.; Ahmed, J.; Kojic, A.; Control Systems, IEEE, 2010, 30, 49.

25. Feng, Y.; Xue, C.; Han, Q.; Han, F.; and Du. J.; IEEE Trans. on Industrial Electronics, online May 2019.

26. Feng, Y.; Han, F.; Yu. X.; Automatica, 2014, 50, 1310.

27. Duong, T. Q.; J. Power Sources, 2000, 89, 244.

28. The CALCE battery group, Available: https://web.calce.umd.edu/batteries/data.htm (Accessed: Jul. 25, 2018). 